\title{
Properties of galactic B[e] supergiants II. HDE 327083
}

\author{
A. S. Miroshnichenko ${ }^{1,2}$, H. Levato ${ }^{3}$, K. S. Bjorkman ${ }^{1}$, and M. Grosso ${ }^{3}$ \\ ${ }^{1}$ Ritter Observatory, Dept. of Physics and Astronomy, University of Toledo, Toledo, OH 43606-3390, USA \\ 2 Central Astronomical Observatory of the Russian Academy of Sciences at Pulkovo, 196140 Saint-Petersburg, Russia \\ 3 Complejo Astronómico El Leoncito (CASLEO), Casilla de Correo 467, 5400 San Juan, Argentina
}

Received 27 February 2003 / Accepted 16 May 2003

\begin{abstract}
The emission-line object HDE 327083 has long been considered to be one of the most luminous stars in the Milky Way. Nevertheless, no reliable physical parameters have been published for it. Our high-resolution optical spectroscopy revealed the presence of photospheric lines of a cool luminous companion. We detected significant antiphased radial velocity variations of the emission and absorption lines. The data obtained are still insufficient to derive a reliable orbital solution; however, the orbital period is most likely of the order of 6 months. We conclude that HDE 327083 is a binary system consisting of an early B-type primary and early F-type secondary, with luminosities $\log L / L_{\odot}=5.0 \pm 0.4$ and $4.2 \pm 0.4$, respectively, and whose orbital plane is viewed nearly edge-on. We also obtained new multicolour optical and infrared photometry of HDE 327083. From both the photometric and spectroscopic data, we found that the system is located at a distance of $1.5 \pm 0.5 \mathrm{kpc}$. Most of the circumstellar gas seems to be orbiting the primary and is distributed in a mildly flattened envelope with a height scale and velocity decreasing outward from the star. We suggest that HDE 327083 represents an advanced evolutionary stage of a $\beta$ Lyrae type binary.
\end{abstract}

Key words. stars: emission-line, Be - stars: individual: HDE 327083 - techniques: spectroscopic - techniques: polarimetric

\section{Introduction}

$\mathrm{B}[\mathrm{e}]$ stars are a group of early-type galactic objects that show forbidden emission lines and near-IR excess due to circumstellar (CS) dust. Discovered in the 1970's (Allen \& Swings 1976), they have not been intensively studied until recently, mostly due to their relative faintness and the inhomogeneity of their intrinsic parameters. The advent of high-resolution spectroscopy revealed new, sometimes unexpected, features of these objects and provided a tool for refining their properties. One of the subgroups of $\mathrm{B}[\mathrm{e}]$ stars contains high-luminosity objects, whose fundamental parameters, nature and evolutionary state are still poorly known due to uncertainties in the distance determination and complexity of the observed features. Similar objects, B[e] supergiants, were found in the Magellanic Clouds (Zickgraf et al. 1985), where they are among the brightest stars, confirming their high luminosity. They show very strong emission-line spectra indicating a large amount of CS matter in the immediate vicinity of the radiation source. Their spectral line profiles suggest the presence of a fast and hot polar wind (UV lines) and a slower and cooler equatorial one (optical lines). B[e] supergiants may be LBV precursors (e.g., Conti 1997), which makes

Send offprint requests to: A. S. Miroshnichenko, e-mail: anatoly@physics.utoledo.edu their investigation relevant for our understanding of high-mass stellar evolution.

At the same time, several galactic non-luminous B[e] stars share the properties of the Magellanic Clouds B[e] supergiants (see Miroshnichenko et al. 2002a for a recent review). This makes qualitative classification of $\mathrm{B}[\mathrm{e}]$ stars ambiguous and calls for quantitative studies, which require different techniques and methods of analysis. This paper continues our series of studies of galactic B[e] stars that have been classified as supergiants. In the first paper (Miroshnichenko et al. 2002b) we presented the results of high-resolution spectroscopy of CI Cam, a binary system containing a $\mathrm{B}[\mathrm{e}]$ star and a compact object (neutron star or black hole, e.g. Robinson et al. 2002). We did not find convincing evidence for a large distance and, hence, high luminosity of the $\mathrm{B}[\mathrm{e}]$ companion in CI Cam, and we suggested some observational tests to further constrain the objects parameters.

In this paper we report the results of our spectroscopic and photometric study of a southern object HDE 327083. This object was not included in the original list of $\mathrm{B}[\mathrm{e}]$ stars. Attention to this object was drawn by Carlson \& Henize (1979), who reported a strong emission-line spectrum containing lines of $\mathrm{H} \mathrm{I}, \mathrm{He} \mathrm{I}$, and Fe II. Optical $U B V$ photometry (Kozok 1985) showed that it was a very reddened early B-type star $(E(B-V) \sim 1.9 \mathrm{mag})$. Near-IR spectroscopy 
(Whitelock et al. 1983; McGregor et al. 1988) revealed a strong near-IR excess and CO emission features at $2.3 \mu \mathrm{m}$. The IRAS satellite (Olnon et al. 1986) detected a strong mid-IR flux from HDE 327083, with a steep decrease towards longer wavelengths, and a featureless spectrum in the $10 \mu \mathrm{m}$ region. Although no solid-state features have been detected, the IR excess is too strong to be attributed to CS gas alone and implies the presence of CS dust, which makes the object similar to "Be stars with warm dust", a subgroup of galactic B[e] stars (Miroshnichenko et al. 2002a).

Optical spectroscopic observations of selected regions containing some emission lines and interstellar features by Lopes et al. (1992) confirmed the P Cyg-type profile of the hydrogen and singly ionized iron lines earlier reported by Henize (1952) and Carlson \& Henize (1979). Lopes et al. also estimated the distance $(D)$ towards HDE 327083 based on the strength of the interstellar sodium D-lines $(D(\mathrm{kpc})=2 E W(\AA)$, where $E W$ is the average of the equivalent widths of the $\mathrm{D}_{1}(\lambda 5895 \AA)$ and $\mathrm{D}_{2}(\lambda 5889 \AA)$ lines, Allen 1955). At this distance (5 kpc) the object's luminosity would be very high $\left(M_{V}=-10 \mathrm{mag}\right)$, making it one of the most luminous stars in the Milky Way. These authors also assigned a spectral type of B6 I to the object.

Recently Machado et al. (2001) obtained new spectra of HDE 327083 in the regions of $\mathrm{H} \alpha, \mathrm{H} \beta$, and several Fe II lines and tried to model the Balmer line profiles. They suggested 2 different sets of fundamental parameters for the star based on the line profile fits, although their fits did not closely reproduce the profile shapes. Both models imply a very high luminosity $\left(\gtrsim 10^{5} L_{\odot}\right)$ and mass loss rate $\left(\gtrsim 10^{-5} M_{\odot} \mathrm{yr}^{-1}\right)$. The assumed radiation source was a hot star with an effective temperature $\left(T_{\text {eff }}\right)$ of either $9000 \mathrm{~K}$ or $19000 \mathrm{~K}$.

These studies indicate that HDE 327083 is a complex object whose physical characteristics are not well constrained. This prompted us to undertake a new study of the object using both spectroscopic and photometric technique. The observations are described in Sect. 2, our new results in Sect. 3, analysis of the observed properties in Sect. 4, and our conclusions in Sect. 5.

\section{Observations}

The spectroscopic observations were obtained at the 2.1-m telescope of the Complejo Astronómico El Leoncito (Argentina) with the échelle-spectrograph REOSC, mounted in the Nasmyth-2 focus and equipped with a $2 \mathrm{~K} \times 2 \mathrm{~K}$ CCDchip. This setup allowed us to achieve a spectral resolving power $R \sim 15000$. Additionally, 1 spectrum was obtained at the 2.1-m Otto Struve telescope of the McDonald Observatory (Mt. Locke, Texas, USA) with the Sandiford échelle-spectrometer (McCarthy et al. 1993) with a $R \sim 60000$. A $1200 \times 400$ pixel CCD was used. The spectrum was taken at a large air mass of 4.5 due to the southern location of the object. As a result, it was a bit noisy, but the line characteristics were consistent with those from the Leoncito spectra.

There are small gaps between the spectral orders in the Leoncito spectra, while all the orders of the McDonald spectrum are overlapped. The log of the spectroscopic observations
Table 1. Log of spectroscopic observations of HDE 327083.

\begin{tabular}{lccc}
\hline \hline Date & $\begin{array}{c}\text { JD } \\
2450000+\end{array}$ & $\begin{array}{c}\text { Exp.time } \\
\mathrm{s}\end{array}$ & $\begin{array}{c}\text { Sp. region } \\
\AA\end{array}$ \\
\hline $2000 / 07 / 19$ & 1744.63 & 1800 & $3880-5845$ \\
$2000 / 07 / 21$ & 1746.58 & 300 & $5300-8450$ \\
$2002 / 03 / 03$ & 2336.83 & 900 & $5595-8740$ \\
$2002 / 05 / 23$ & 2417.63 & 1000 & $5710-8750$ \\
$2002 / 05 / 24$ & 2418.71 & 1000 & $5670-8740$ \\
$2002 / 06 / 23$ & 2448.59 & 3600 & $5820-9020$ \\
$2002 / 06 / 24$ & 2449.74 & 3600 & $5820-9020$ \\
$2002 / 06 / 25$ & 2450.70 & 3600 & $5820-9020$ \\
$2002 / 09 / 25^{\text {a }}$ & 2543.58 & 1200 & $5430-6735$ \\
\hline Obtained at the McDonald Observatory. &
\end{tabular}

is presented in Table 1 . The standard data reduction was performed in IRAF $^{1}$.

The optical (in the Johnson-Cousins system) and nearIR broadband photometric observations of HDE 327083 were obtained quasi-simultaneously on 1997 March 2 and 3 at the South-African Astronomical Observatory (SAAO). The 0.75-meter telescope with a single-element InSb photometer was used in the near-IR, while the 0.5-meter telescope with a GaAs photometer was used in the optical region. Additional IR observations were obtained on 2000 February 25 at Mauna Kea with the 3-meter NASA Infrared Telescope Facility (IRTF) and a single-element gallium-doped germanium bolometer. Our photometric data are presented in Table 2. The accuracy is about 0.01 mag. and 0.03 mag. for the SAAO optical and IR data, respectively, and 0.05 mag. for the IRTF data. A number of photometric standard stars were observed during the same nights at both observatories for calibration.

\section{Results}

\subsection{The spectrum}

The previous spectroscopic observations of HDE 327083 discussed in Sect. 1 were performed in selected narrow regions and focused on emission lines. In contrast, we studied a much broader spectral region and tried to find every possible spectral feature. The emission line content and their profile shapes were found to be similar to those reported in the literature. HDE 327083 clearly shows emission lines of $\mathrm{H} \mathrm{I}$, He I, and Fe II, most of which have P Cyg type profiles. The $\mathrm{H} \alpha$ line has a slightly variable double-peaked profile with the peak intensity ratio $V / R \sim 0.1$. The central depression is narrow with the minimum intensity below continuum at highresolution (Fig. 1a). The He I lines display a weak emission component, which almost vanishes at a low signal-to-noise ratio (SNR). Their absorption components reach the continuum at a heliocentric radial velocity $(H R V)$ of $\sim-400 \mathrm{~km} \mathrm{~s}^{-1}$ and have a mean $H R V$ of $\sim-200 \mathrm{~km} \mathrm{~s}^{-1}$ (derived by fitting the

\footnotetext{
${ }^{1}$ IRAF is distributed by the National Optical Astronomy Observatories, which are operated by the Association of Universities for Research in Astronomy, Inc., under contract with the National Science Foundation.
} 
Table 2. New photometry of HDE 327083.

\begin{tabular}{rlllllllllll}
\hline \hline JD & $V$ & $U-B$ & $B-V$ & $V-R_{\mathrm{c}}$ & $V-I_{\mathrm{c}}$ & $J$ & $H$ & $K$ & $L$ & $M$ & $N$ \\
$2450000+$ & & & & & & & & & & & \\
\hline 510.62 & 9.68 & 0.52 & 1.65 & 1.14 & 2.25 & 5.65 & 4.59 & 3.67 & 2.44 & & \\
511.61 & 9.65 & 0.50 & 1.65 & 1.14 & 2.26 & 5.62 & 4.55 & 3.63 & 2.38 & & \\
1599.14 & & & & & & & & 3.76 & 2.37 & 1.69 & 0.93 \\
\hline
\end{tabular}

component with a Gaussian), which corresponds to that of the $\mathrm{H} \alpha$ blue emission peak. In the high SNR spectra we also detected the Si II 6347 and $6371 \AA$ lines with both an emission and absorption component (Fig. 2b); however, these are not parts of a P Cyg type profile (see below).

As in the spectra published by Lopes et al. (1992) and Machado et al. (2001), we detected a number of Fe II lines with P Cyg type profiles. Their absorption components are strong in lines blueward of $\lambda \sim 5300 \AA$, while they are weak or undetectable in redder lines. The emission components are definitely double-peaked. The split is barely seen in the red Leoncito spectra (see Fig. 2), but it becomes obvious in the blue Leoncito and the McDonald spectrum (Fig. 3).

The only detection of the [Fe II] line at $5158 \AA$ in the blue Leoncito spectrum of 2000 July 19. It was also previously reported by Machado et al. (2001). The forbidden [N II] line at $5755 \AA$ and the [O I] lines at 6300 and $6363 \AA$ are seen in some of our spectra, but most likely have telluric nature. They are unshifted from laboratory wavelengths and have a narrower width $\left(8 \mathrm{~km} \mathrm{~s}^{-1}\right.$ at half maximum in the Mcdonald spectrum) than the other emission features. The forbidden [N II] lines at 6548 and $6583 \AA$ are extremely weak and hardly noticeable at $R=15000$. The [N II] line at $6548 \AA$ was detected in the Mcdonald spectrum with the same width as the other forbidden lines. This suggests that the density virtually everywhere in the gaseous envelope of HDE 327083 is above $10^{6} \mathrm{~cm}^{-3}$ (Osterbrock 1989). It may be even above $10^{7} \mathrm{~cm}^{-3}$ because of the absence of the [S II] lines at $6312 \AA$. However, the latter could be due to an insufficiently high $T_{\text {eff }}$ of the radiation source responsible for the emission line spectrum. Since we do not see $\mathrm{He}$ II lines in emission and the He I lines have weak emission components, we can place an upper limit of B2 on the hot star spectral type (e.g., Miroshnichenko 1996).

The Leoncito spectra show the IR triplet of Ca II at 8498 , 8542, and $8662 \AA$. These lines have strong emission and weak absorption components. In some of our spectra the emission components are double-peaked, with a peak separation of $\sim 75 \mathrm{~km} \mathrm{~s}^{-1}$ (Fig. 1c). The absorption components have similar shapes to those of the He I lines and are due to the Paschen lines (P12, P13, and P15), which have much weaker emission components than those of the Ca II lines. For example, the $\lambda 8498 \AA$ calcium line is fully located inside the P12 line absoprtion component.

The sodium D-lines at 5889 and $5995 \AA$ have very weak emission components which are not noticeable in every spectrum. At $R=15000$ two absorption components are seen. One of them is deep and narrow, has its position unchanged in all the spectra $\left(H R V=-25 \mathrm{~km} \mathrm{~s}^{-1}\right)$, and is certainly of interstellar origin. At higher resolution it splits into 2 components at $H R V \mathrm{~s}$ of $-11 \mathrm{~km} \mathrm{~s}^{-1}$ and $-35 \mathrm{~km} \mathrm{~s}^{-1}$. The red component seems to be formed in the local spiral arm and is saturated, while the blue one originates in the Sagittarius arm and is close to saturation (Fig. 1b). There is also another absorption component of the D-lines, which is weak and broad and has a $H R V$ of $\sim-130 \mathrm{~km} \mathrm{~s}^{-1}$. This component is most likely CS, with the position close to that of the $\mathrm{H} \alpha$ central depression.

Strong diffuse interstellar bands (DIB) are seen in our spectra. They have single-peaked and nearly gaussian profiles with a mean $H R V$ of $-6 \pm 1 \mathrm{~km} \mathrm{~s}^{-1}$, averaged over all our spectra. There is a sign of a split in the strongest DIBs at 5780 and $5797 \AA$ in the McDonald spectrum, where the red component is much stronger than the blue one. This may be due to the components formation in different spiral arms, similar to that in the Na I D-lines. At the same time, the other DIBs detected in this spectrum $(6269,6379,6613,6660$, and $6699 \AA$ A) do not show such a split. Furthermore, a low SNR in the continuum of this spectrum $(\sim 30)$ makes this split uncertain.

Photospheric lines have not been reported previously for HDE 327083. We also do not find those appropriate for a hot star in our spectra. At the same time, we detected a large number of relatively weak absorption lines, most of which were identified with neutral metals (see Table 3 and Figs. 2-4). Moreover, we found that the mean $H R V$ of these lines varies with time (see Fig. 5), and its amplitude $\left(\sim 80 \mathrm{~km} \mathrm{~s}^{-1}\right)$ is much higher than its statistical accuracy $\left(\sim 5 \mathrm{~km} \mathrm{~s}^{-1}\right.$, Table 4$)$.

The absorption components of the Si II lines have the same $H R V \mathrm{~s}$ as those of the other absorption lines, suggesting that they belong to the atmosphere of a cool companion, while the emission components may be formed in the CS envelope. The presence of the Si II lines in the spectrum of the cool companion sets a lower limit on its $T_{\text {eff }}$, because these lines are not seen in K-type stars. Moreover, in G-type stars these lines are weaker than others in this spectral region (e.g., Fe I (Mult. 13) $\lambda 6358 \AA$ ). A comparison of the spectra of HDE 327083 and MWC 623, a B[e] binary with a K-type secondary companion (Zickgraf 2001), shows that HDE 327083 has a much poorer absorption line content than MWC 623 (Fig. 4). Therefore, the cool companion most likely has an A- or F-type.

Photospheric absorption lines were detected in our spectra redward of $\sim 4700 \AA$. We do not see them in the bluer regions for the following reasons. First, our only blue spectrum has a low SNR, and, second, the hot companion's continuum becomes dominant in this spectral region. However, the observed absorption-line spectrum suggests that the companions are almost equally bright in the $V$-band. Indeed, the comparison of the secondary's spectrum (which was obtained by 

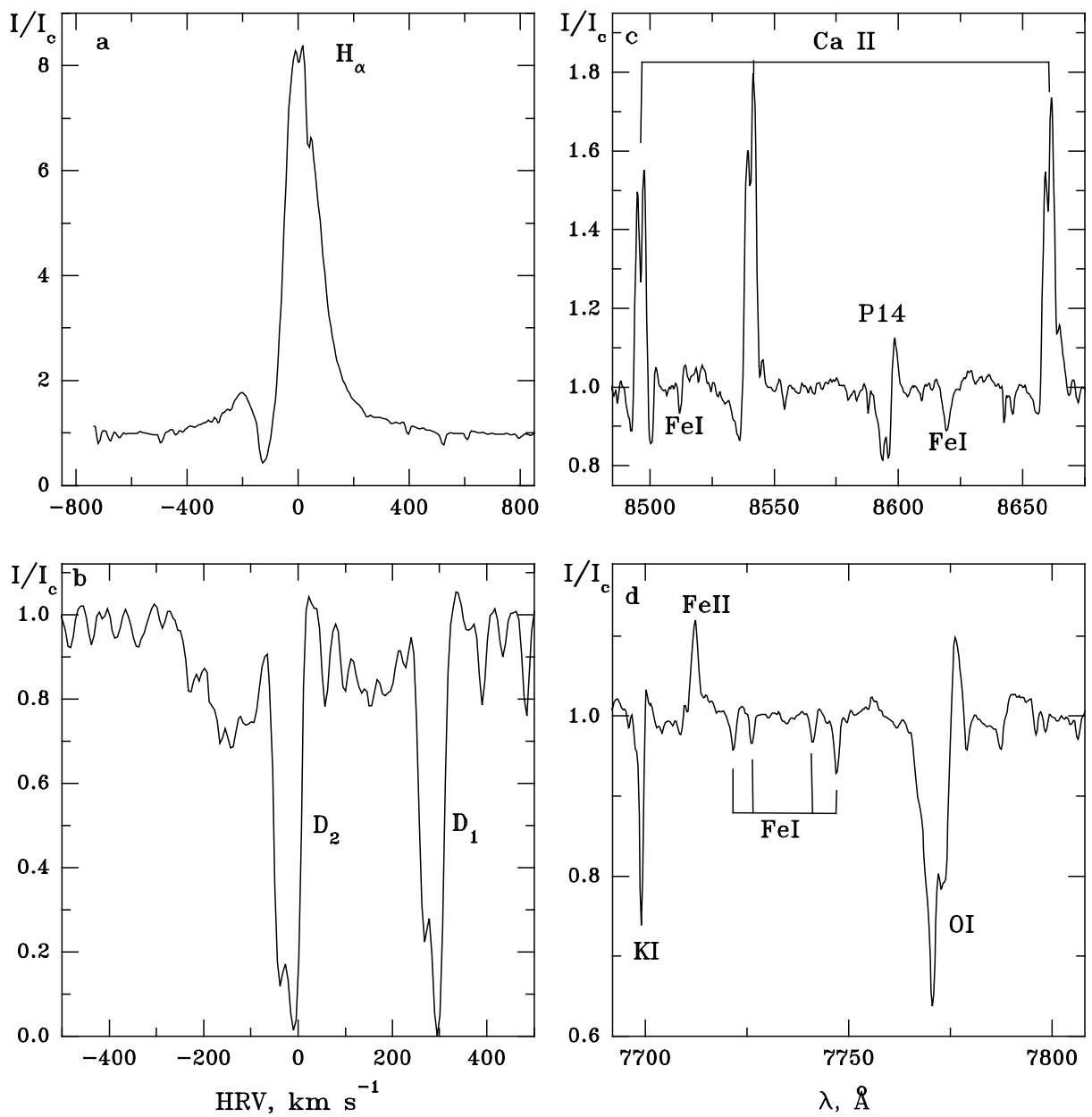

Fig. 1. Portions of the spectrum of HDE 327083. Panels a) and b) show the H $\alpha$ and Na I D lines from the McDonald data. All the weak absorption features are telluric. The intensities are in continuum units, and the heliocentric radial velocities are in $\mathrm{km} \mathrm{s}^{-1}$. Panels $\mathbf{c}$ ) and d) show the Ca II and O I IR triplets in the Leoncito spectra. Some photospheric Fe I lines of the secondary and the interstellar K I $\lambda 7699 \AA$ A line are also shown. The intensities are in continuum units, and the heliocentric wavelengths are in $\AA$.
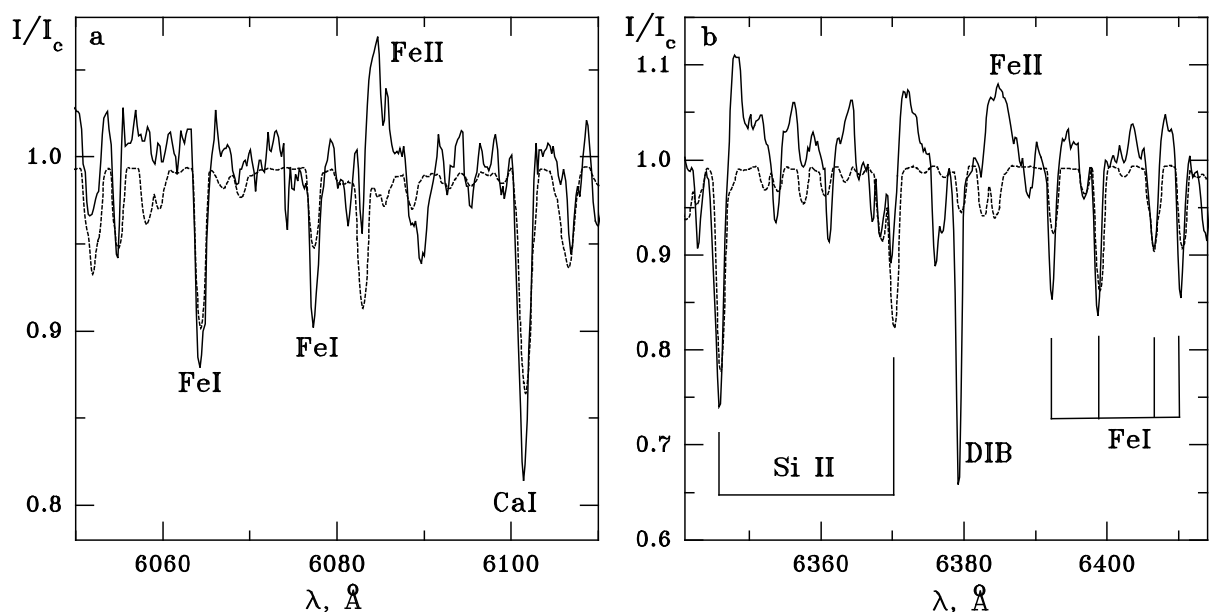

Fig. 2. Portions of the 2002 June 25 Leoncito spectrum containing the secondary's absorption lines. The object's spectrum with the primary's continuum subtracted is shown by solid lines. The theoretical spectrum for $T_{\text {eff }}=7000 \mathrm{~K}, \log g=2.0$, and $v \sin i=40 \mathrm{~km} \mathrm{~s}^{-1}$ calculated using the Synspec code (Hubeny et al. 1995) is shown by dotted lines. The intensities are in continuum units, and the heliocentric wavelengths are in $\AA$.

subtraction of the featureless primary's continuum from the normalized observed spectrum) with theoretical spectra shows a very good agreement for $T_{\text {eff }}=7000 \mathrm{~K}, \log g=2.0$, and $v \sin i=30-40 \mathrm{~km} \mathrm{~s}^{-1}$ (see Fig. 2). The continuum intensities 
Table 3. Absorption lines of the secondary companion in the spectrum of HDE 327083.

\begin{tabular}{|c|c|c|c|c|c|c|c|c|c|c|c|}
\hline$\lambda_{\text {lab }}$ & ID & $E W, \AA$ & $I / I_{\mathrm{c}}$ & $\lambda_{\text {lab }}$ & ID & $E W, \AA$ & $I / I_{\mathrm{c}}$ & $\lambda_{\mathrm{lab}}$ & ID & $E W, \AA$ & $I / I_{\mathrm{c}}$ \\
\hline (1) & (2) & (3) & (4) & (1) & (2) & (3) & (4) & (1) & (2) & (3) & (4) \\
\hline 4710.19 & TiI (75) & 0.08 & 0.89 & 5952.75 & FeI (959) & 0.04 & 0.96 & 6419.98 & FeI (1258) & 0.08 & 0.91 \\
\hline 4779.99 & TiII(92) & 0.11 & 0.83 & 6003.03 & FeI (959) & 0.03 & 0.96 & 6421.36 & FeI (111) & 0.11 & 0.90 \\
\hline 4783.42 & MnI (16) & 0.11 & 0.87 & 6008.58 & FeI (982) & 0.06 & 0.95 & 6439.07 & CaI (18) & 0.21 & 0.82 \\
\hline 4805.11 & TiII(92) & 0.09 & 0.85 & 6021.80 & MnI (27) & 0.04 & 0.96 & 6449.81 & CaI (19) & 0.13 & 0.92 \\
\hline 5328.04 & FeI (37) & 0.21 & 0.82 & 6020.17 & FeI (1178) & 0.06 & 0.94 & 6462.57 & CaI (18) & 0.16 & 0.86 \\
\hline 5371.49 & FeI (15) & 0.10 & 0.84 & 6024.07 & FeI (1178) & 0.07 & 0.92 & 6471.66 & CaI (18) & 0.05 & 0.95 \\
\hline 5404.14 & FeI (1165) & 0.11 & 0.86 & 6041.93 & S I (10) & 0.06 & 0.93 & 6604.60 & $\operatorname{ScII}(19)$ & 0.05 & 0.96 \\
\hline 5405.78 & FeI (15) & 0.12 & 0.85 & 6046.04 & S I (10) & 0.03 & 0.97 & 6633.76 & FeI (1197) & 0.10 & 0.94 \\
\hline 5455.61 & FeI (15) & 0.13 & 0.84 & 6055.99 & FeI (1259) & 0.03 & 0.96 & 6717.69 & CaI (32) & 0.06 & 0.94 \\
\hline 5594.47 & $\mathrm{CaI}(21)$ & 0.11 & 0.89 & 6065.81 & FeI $(581)$ & 0.07 & 0.92 & 6743.58 & S I (8) & 0.06 & 0.93 \\
\hline 5598.49 & CaI (21) & 0.12 & 0.86 & 6078.50 & FeI (1259) & 0.13 & 0.89 & 6748.79 & S I (8) & 0.04 & 0.96 \\
\hline 5615.65 & FeI (686) & 0.17 & 0.85 & 6102.72 & $\mathrm{CaI}(3)$ & 0.20 & 0.86 & 6757.16 & S I (8) & 0.04 & 0.95 \\
\hline 5620.53 & FeI (1061) & 0.03 & 0.94 & 6122.22 & CaI (3) & 0.11 & 0.87 & 6855.18 & FeI (1195) & 0.05 & 0.96 \\
\hline 5624.61 & V I (37) & 0.11 & 0.88 & 6141.73 & FeI (816) & 0.17 & 0.84 & 6945.21 & FeI (111) & 0.09 & 0.93 \\
\hline 5641.46 & FeI (1087) & 0.14 & 0.89 & 6162.17 & CaI (3) & 0.13 & 0.88 & 7148.15 & CaI (30) & 0.07 & 0.92 \\
\hline 5658.42 & FeI (686) & 0.18 & 0.86 & 6191.56 & FeI (169) & 0.10 & 0.90 & 7405.85 & SiI (23) & 0.09 & 0.94 \\
\hline 5662.53 & FeI (1087) & 0.10 & 0.89 & 6230.73 & FeI (207) & 0.09 & 0.90 & 7409.11 & SiI (23) & 0.05 & 0.95 \\
\hline 5747.36 & N I (35) & 0.06 & 0.95 & 6244.56 & SiI (27) & 0.24 & 0.91 & 7411.18 & FeI (1077) & 0.03 & 0.97 \\
\hline 5753.14 & FeI (1107) & 0.06 & 0.95 & 6347.09 & $\operatorname{SiII}(2)$ & 0.23 & 0.86 & 7445.78 & FeI (1077) & 0.05 & 0.94 \\
\hline 5762.99 & FeI (1107) & 0.07 & 0.93 & 6371.36 & $\operatorname{SiII}(2)$ & 0.18 & 0.88 & 7495.09 & FeI (1077) & 0.09 & 0.94 \\
\hline 5772.26 & SiI (17) & 0.06 & 0.95 & 6393.61 & FeI (168) & 0.09 & 0.90 & 7511.05 & FeI (1077) & 0.11 & 0.91 \\
\hline 5853.68 & BaII(2) & 0.07 & 0.93 & 6400.01 & FeI (816) & 0.13 & 0.87 & 7998.97 & FeI (1136) & 0.12 & 0.93 \\
\hline 5857.45 & CaI (47) & 0.09 & 0.90 & 6408.03 & FeI (816) & 0.10 & 0.93 & 8046.07 & FeI (1136) & 0.07 & 0.95 \\
\hline 5862.36 & FeI (1180) & 0.04 & 0.95 & 6411.66 & FeI (816) & 0.10 & 0.92 & 8085.20 & FeI (1136) & 0.06 & 0.95 \\
\hline 5948.58 & SiI (16) & 0.11 & 0.91 & & & & & & & & \\
\hline
\end{tabular}

The laboratory wavelength in $\AA$ is listed in Col. 1, the line identification in Col. 2, the line equivalent width averaged in Col. 3, and the line depth in the underlying continuum units in Col. 4. The variations of EW and depth are small and may be attributed mainly to measurement errors.

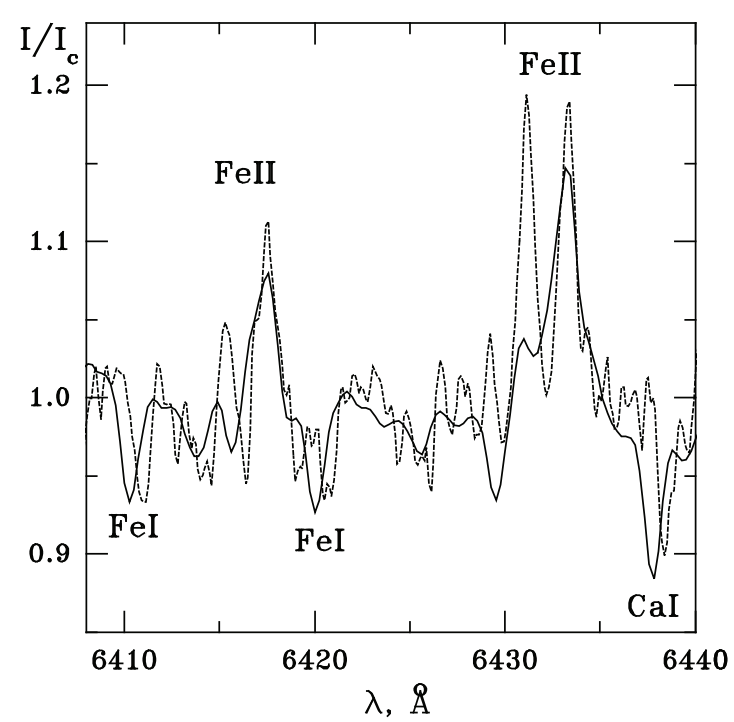

Fig. 3. A portion of the spectrum of HDE 327083 containing Fe II lines. The solid line shows the Leoncito spectrum of 2002 June 25, while the dashed line shows the Mcdonald spectrum of 2002 September 25. The displacement of the photospheric lines is clearly seen. The intensities are in continuum units, and the heliocentric wavelengths are in $\AA$. of the companions were fixed as equal. The estimated secondary's parameters correspond to those of an early F-type supergiant.

One of the prominent spectral features is a strong $\mathrm{O}$ I triplet at 7771-7775 $\AA$ (Fig. 1d). It is usually weak in hot (B-type) and low-luminosity stars, and thus can be attributed to the secondary's atmosphere. According to a recent luminosity calibration by Klochkova et al. (2002), the most luminous stars $\left(M_{V} \sim-9 \pm 1 \mathrm{mag}\right)$ show $E W(\mathrm{O} \mathrm{I})=2.0-2.7 \AA$. The observed $E W(\mathrm{O}$ I) in the spectrum of HDE 327083 is $1.7 \pm 0.1 \AA$, which becomes $3.2 \pm 0.3 \AA$ after subtracting a featureless contribution of the primary using a reasonable component brightness ratio at the triplet's wavelength $(0.8 \pm 0.2)$. This result would suggest that the primary's luminosity is above the Eddington level, which seems unreliable. Moreover, in our spectrum of CPD $-52^{\circ} 9243$, a B [e] star with very similar observed features, the $E W(\mathrm{O}$ I $)=4.8 \AA$. Thus, $C S$ matter may contribute to the observed strength of the triplet, restricting reliable luminosity estimates based on this $E W$ to normal stars and objects with weak emission-line spectra.

The emission line components seem to change their $H R V \mathrm{~s}$ (measured by matching the original and mirrored line profiles) in antiphase to those of the absorption lines (see Figs. 5-6). 


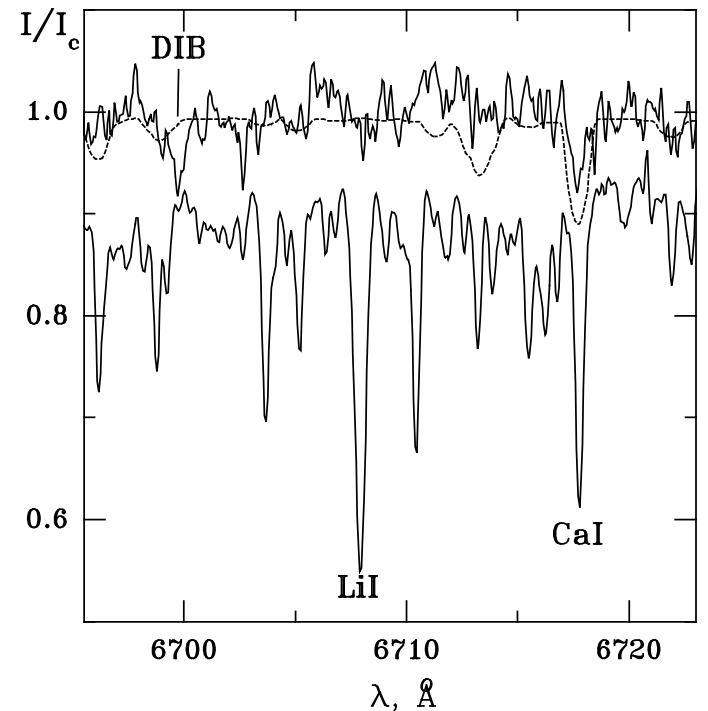

Fig. 4. Portions of the spectra of HDE 327083 (upper solid line) and MWC 623 (lower solid line) obtained at the McDonald Observatory. The dashed line represents the theoretical spectrum with the same parameters as in Fig. 2. Most of the unlabeled lines in the spectrum of MWC 623 are those of Fe I. The intensities are in continuum units, and the heliocentric wavelengths are in $\AA$.

However, due to the weak and broad nature of most of the emission lines, their $H R V \mathrm{~s}$ are less reliable. Their profiles may also be affected by the envelope's kinematics. This is why we consider the emission-line $H R V \mathrm{~s}$, shown in Fig. 5 and listed in Table 3, to have a lower statistical weight than those of the photospheric lines. As seen in Fig. 5, their $H R V$ s do not closely agree with the solution obtained for the photospheric lines.

The $\mathrm{H} \alpha$ line was saturated in half of our spectra, because we tried to achieve a high SNR in the continuum. In those spectra where the observed $\mathrm{H} \alpha$ profile was not affected by the detector non-linearity, the positions of the emission peaks and central depression were stable within $\pm 10 \mathrm{~km} \mathrm{~s}^{-1}$.

The described behaviour caused us to suspect that the pure absorption-line spectrum belongs to a cooler star, the secondary companion of the B-type primary. The possible parameters of the binary system are discussed in Sect. 4.

\subsection{Spectral energy distribution}

Our optical photometry obtained in 1997 showed that HDE 327083 was about 0.2 mag fainter in the $V$-band with respect to the $1979 / 80$ data of Kozok (1985), while the $U-B$ and $B-V$ colour-indices were very close in both data sets. The broadband IR photometric data for HDE 327083 were obtained by us for the first time. Earlier McGregor et al. (1988) published continuum fluxes in the $H$ and $K$ bands (13.1 and $21.8 \mathrm{Jy}$, respectively) measured from their absolutely calibrated low-resolution spectra. These fluxes are in good agreement with our results. Also our result for the $N$ band $(10.4 \mu \mathrm{m})$ obtained in 2000 turned out to be close to the IRAS $12-\mu \mathrm{m}$ flux measured in 1983 and to the MSX A-band $(8.28 \mu \mathrm{m})$ flux measured in 1996. The spectral energy distribution (SED) of HDE 327083 corrected for the reddening is shown in Fig. 7.
Table 4. Radial velocities of the spectral lines of HDE 327083.

\begin{tabular}{lrrcccc}
\hline \hline Date & $\begin{array}{c}\text { Photosph. } \\
\mathrm{km} \mathrm{s}^{-1}\end{array}$ & $N$ & $\begin{array}{c}\text { Fe II } \\
\mathrm{km} \mathrm{s}^{-1}\end{array}$ & $N$ & $\begin{array}{c}\text { DIB } \\
\mathrm{km} \mathrm{s}^{-1}\end{array}$ & $N$ \\
\hline $2000 / 07 / 19$ & $-5 \pm 5$ & 9 & & & & \\
$2000 / 07 / 21$ & $-1 \pm 7$ & 12 & $-15 \pm 1$ & 4 & $-3 \pm 2$ & 3 \\
$2002 / 03 / 03$ & $-56 \pm 9$ & 37 & $+25 \pm 6$ & 9 & $-6 \pm 3$ & 5 \\
$2002 / 05 / 23$ & $+13 \pm 5$ & 13 & $-82 \pm 8$ & 4 & $-4 \pm 2$ & 3 \\
$2002 / 05 / 24$ & $+13 \pm 4$ & 24 & $-79 \pm 6$ & 3 & $-5 \pm 2$ & 3 \\
$2002 / 06 / 23$ & $-62 \pm 5$ & 23 & $+23 \pm 1$ & 2 & $-7 \pm 2$ & 3 \\
$2002 / 06 / 24$ & $-64 \pm 5$ & 45 & $+21 \pm 5$ & 5 & $-5 \pm 2$ & 5 \\
$2002 / 06 / 25$ & $-67 \pm 5$ & 48 & $+22 \pm 4$ & 7 & $-6 \pm 2$ & 4 \\
$2002 / 09 / 25$ & $-36 \pm 5$ & 14 & $-27 \pm 2$ & 3 & $-8 \pm 3$ & 7
\end{tabular}

The mean $H R V$ of the photospheric lines are listed in Col. 2, those of the emission peaks of the Fe II lines in Col. 4, and those of the DIBs in Col. 6. The number of lines used for the averaging are listed in Cols. 3, 5, and 7, respectively. The DIBs $H R V$ measurements show the wavelength scale stability.

The de-reddening procedure and the SED features are further discussed in Sect. 4.

Thus, the object may be photometrically variable in the optical region. The IR brightness seems to be more stable. However, the small amount of data does not allow us to draw a more definite conclusion about the variability level.

In general, the IR excess of HDE 327083 is similar to those of other Be stars with warm dust (e.g., Miroshnichenko et al. 2001). It comprises about 1 per cent of the total illuminating radiation, which corresponds to an optical depth of $\sim 0.01$ if the dust is spherically distributed around the system. However, this distribution seems to be unlikely for a supposedly evolved binary. Furthermore, the IR spectrum in the $10 \mu \mathrm{m}$ region is essentially flat, implying either a moderate optical depth or the presence of amorphous carbon as the dominant dusty component. The former case is more attractive as the dust was probably formed in the system, which has a preferential plane for CS matter concentration. The latter case implies that at least one of the companions is so evolved that carbon from the stellar interiors has reached beyond its surface. Since the spectral line content of HDE 327083 does not show an obvious CNO overabundance, it is more likely that the dusty envelope has a chemical composition similar to the IS. This suggestion is consistent with the results of our SED analysis discussed below in Sect. 4.1.

The steep decrease of the IR flux longward of $\sim 10 \mu \mathrm{m}$ implies that the dusty envelope is compact. In turn, this might imply that it was formed recently, so that it is still close to the illuminating sources. The dust might have been formed during a phase of a rapid mass exchange, which is expected in many binary systems.

\section{Discussion}

We now discuss the findings described above, derive new estimates for the object's parameters, and suggest follow-up 


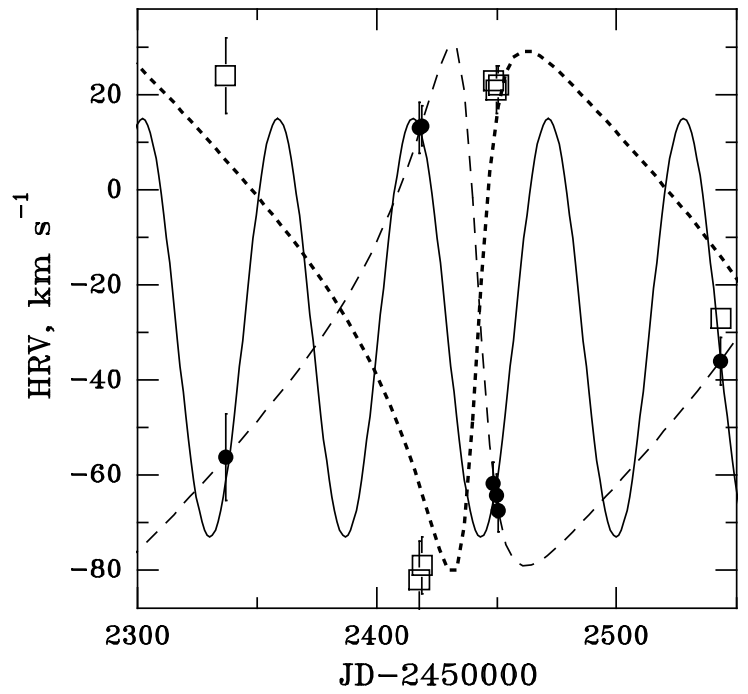

Fig. 5. The radial velocity curve for HDE 327083. The average $H R V$ of the photospheric lines measured in different spectra are shown by filled circles. The $H R V$ of the 2000 Leoncito spectra are not shown to provide a better scale for visual comparison of the data and calculations. The lines represent calculated $R V$ curves for the following parameters: $P_{\text {orb }}=56.5$ days, $K_{2}=45 \mathrm{~km} \mathrm{~s}^{-1}, e=0$ (solid line) and $P_{\text {orb }}=173.5$ days, $K_{2}=55 \mathrm{~km} \mathrm{~s}^{-1}, e=0.57, \omega=66^{\circ}$ (dashed line). The emission line mean $H R V$ derived from our Fe II line data are shown by open squares. The dotted line represents the calculated curve for the primary corresponding to the same eccentric solution as for the secondary and a mass ratio of 1 .

observations to further refine our understanding of its nature and evolutionary state.

\subsection{Reddening, distance and luminosity}

The optical colour-indices of HDE 327083 imply a large reddening which is supported by the strong interstellar (IS) lines and DIBs. At least a part of it may be CS. However as we learned studying other Be stars with warm dust, the CS part of the reddening is usually small, and relationships between the IS extinction and colour-excesses can be applied to unveil the intrinsic SED of these objects (e.g., Miroshnichenko et al. 2000).

Since the IS extinction law varies within the galactic plane, we collected published multicolour photometric data for stars projectionally close to HDE 327083 . These data allowed us to constrain the IS extinction law in the object's direction. In particular, we found that the total-to-selective extinction ratio $R=A_{V} / E(B-V)$ is close to the mean galactic value of 3.1. The colour-excess ratios were found to be as follows: $E(U-B) / E(B-V)=0.83, E\left(V-R_{\mathrm{c}}\right) / E(B-V)=0.65$, $E\left(V-I_{\mathrm{c}}\right) / E(B-V)=1.25, E(V-J) / E(B-V)=2.20$, and $E(V-K) / E(B-V)=2.70$. This is in agreement with the results of He et al. (1995), who used the same data for the stars of our sample as part of their study of the IS extinction in the southern Milky Way.

Application of the $E(U-B) / E(B-V)$ ratio to HDE 327083 gives a reddening independent parameter

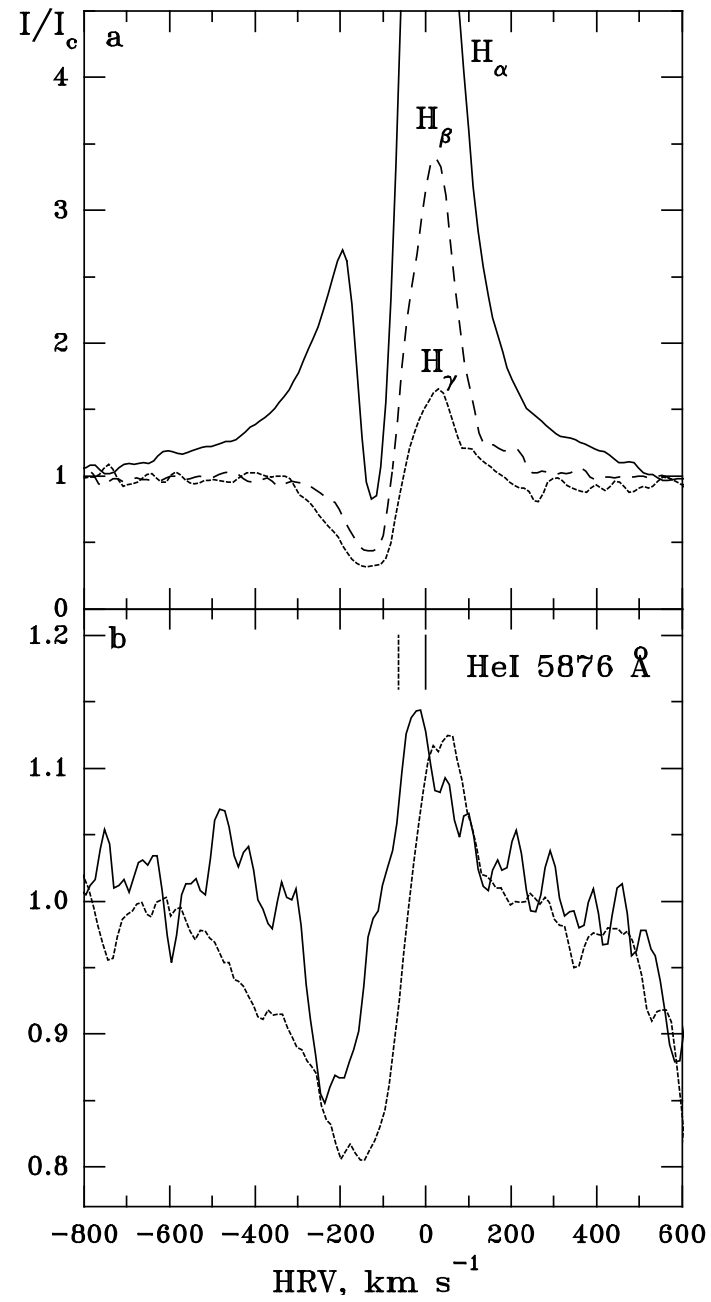

Fig. 6. Emission line profiles in the Leoncito spectra of HDE 327083. a) The Balmer lines. The $\mathrm{H} \alpha$ line from the spectrum of 2002 May 23 is shown by the solid line, the $\mathrm{H} \beta$ and $\mathrm{H} \gamma$ line from the spectrum of 2000 July 21 are shown by the dashed and dotted line, respectively. b) The He I line from the spectrum of 2000 July 19 is shown by the solid line, and that from the spectrum of 2002 June 24 is shown by the dotted line. The short solid and dotted vertical lines mark the mean photospheric line positions on 2000 July 19 and 2002 June 24, respectively. The intensities are in continuum units, and the $H R V$ are in $\mathrm{km} \mathrm{s}^{-1}$.

$Q_{U B V}=(U-B)-0.83(B-V)=-0.86$, which corresponds to a spectral type of B1/2 I or B2/3 V (Strajzhys 1977). These estimates agree with the emission line content discussed in Sect. 3.1. The companion's spectral types and equal brightnesses give a total (IS $+\mathrm{CS})$ colour-excess $E(B-V)_{\text {tot }}=$ $1.8 \mathrm{mag}$ and an extinction $A_{V}^{\text {tot }}=5.6 \mathrm{mag}$. The DIBs strength gives a similar $E(B-V)=1.8 \pm 0.2$ (Herbig 1993), which may also contain some CS contribution. Nevertheless, these results confirm that the hot companion is an early B-type star as follows from the spectroscopic analysis.

The $E\left(V-R_{\mathrm{c}}\right) / E(B-V)$ and $E\left(V-I_{\mathrm{c}}\right) / E(B-V)$ colourexcesses, which were 0.63 and 1.28 , respectively, are also in good agreement with these IS values. This indicates no significant contribution from the cool companion, thus supporting its relatively high $T_{\text {eff. }}$. The colour-excess ratios for the nearIR bands $(J H K L M)$ are higher than the IS ones due to the 


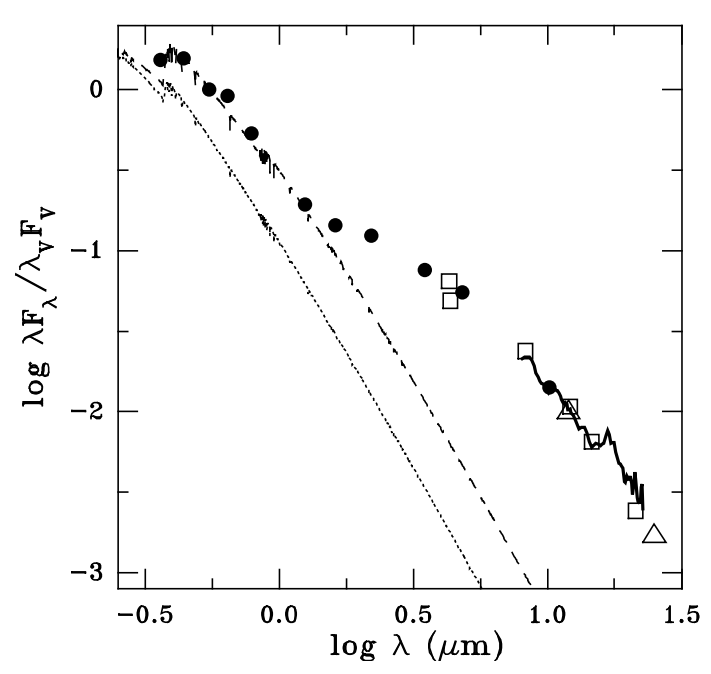

Fig. 7. The spectral energy distribution of HDE 327083. The reddening correction was applied using the extinction values derived from the photometry and adopted spectral types. The IRAS filter photometry is shown by open triangles, the ground-based data by filled circles, the MSX data by open squares, and the IRAS low-resolution spectrum by the solid line. The dotted line represents the Kurucz (1994) theoretical SED for the primary $\left(T_{\text {eff }}=20000 \mathrm{~K}, \log g=3.0\right)$, while the dashed line shows the summed up theoretical SED for the primary and secondary $\left(T_{\text {eff }}=7000 \mathrm{~K}, \log g=2.0\right)$ with a $V$-band flux ratio of 1 . The primary's SED is shifted from the observed one according to the adopted companions' brightness ratio.

radiation of CS dust. The dereddened SED of HDE 327083 is shown in Fig. 7 along with the theoretical SEDs for the companions.

Using the estimated reddening we can put constraints on the location of HDE 327083, whose galactic coordinates $(l=$ $347^{\circ}, b=-1^{\circ}$ ) are close to the direction toward the galactic center. A relationship between the $A_{V}^{\mathrm{IS}}$ and $D$ in a field close to the object's direction $\left(l=346^{\circ}, b=-2^{\circ}\right)$ was constructed by Neckel $\&$ Klare (1980). It shows that $A_{V}^{\text {IS }}$ is about $2.5 \pm$ $1.0 \mathrm{mag}$ at $D=1-4 \mathrm{kpc}$. However, HDE 327083 is closer to the galactic plane than most of this field, which may imply a higher extinction level. Inspection of photometric data for the stars within $1^{\circ}$ of HDE 327083 (mainly from He et al. 1995) shows that $A_{V}$ of 3 and even 5 mag is reached at $D=1.5-3 \mathrm{kpc}$. However, the distance estimates toward these stars are based on spectroscopic parallaxes, which may not be accurate because of possible errors in the luminosity classification.

On the other hand, it is thought that stars are usually confined within spiral arms. According to Rydgren (1974), in addition to the local arm where the Sun is located, two outer spiral arms can be traced in the object's direction. The Sagittarius arm is located at $D=1-2 \mathrm{kpc}$ from the Sun, while the NormaScutum arm is located at $D=3.5-4.5 \mathrm{kpc}$. Optical H II regions at $320^{\circ} \leq l \leq 340^{\circ}$ at $D \geq 3.5 \mathrm{kpc}$ show $R V \mathrm{~s} \leq-45 \mathrm{~km} \mathrm{~s}^{-1}$. We detect no IS feature in the object's spectrum with such a high $R V$. Also the $\mathrm{Na}$ I D-lines only show 2 components (see Sect. 3.1), which most likely belong to the local and the Sagittarius arms. This argument would constrain the location of HDE 327083 within the Sagittarius arm. Thus, the first order estimate for its $D$ is $1.5 \pm 0.5 \mathrm{kpc}$. The strong blue components of the $\mathrm{Na}$ I D-lines favor a location deep within the arm.

The $D$ estimate can then be used to estimate the companions' luminosities. Assuming that they have equal $V$-band brightnesses and taking the mean brightness of the whole system $(V \sim 9.7 \pm 0.1 \mathrm{mag})$, the individual companions turn out to be $V_{1,2} \sim 10.5 \pm 0.1 \mathrm{mag}$. With an $A_{V}=5.6 \pm 0.5$ and a $D=1.5 \pm 0.5 \mathrm{kpc}$ mag, the companions' absolute visual magnitudes are $M_{V}^{1,2}=-5.8 \pm 1.3 \mathrm{mag}$.

The $D=5 \mathrm{kpc}$ derived by Lopes et al. (1992) from the Na I D-line strength is most likely an overestimate, because their interstellar components are saturated (see Fig. 1b). Moreover, their equivalent widths $(E W)$ depend on the spectral resolution. For example, in our Leoncito spectra the sum of the $E W$ s for both lines is $2.3 \AA$, while in the McDonald spectrum it is $2.0 \AA$. Also it is hard to use our $R V$ data to derive a $D$ estimate using the galactic rotation curve. These data do not allow us to determine the systemic $R V$ with confidence.

\subsection{The stellar companions}

Our discovery of the absorption lines of neutral metals in the spectrum of HDE 327083 unambiguously indicates the presence of the secondary companion, which is cooler than the primary responsible for the emission-line spectrum. The secondary should not be much fainter than the primary, since its spectral lines are clearly seen. It does not dominate in the optical region either, as the observed SED is consistent with that of a reddened early B-type star (Sect. 4.1). It seems reasonable that the companions' luminosities and probably masses are comparable. This is followed from comparison of the $R V$ amplitudes of the emission (Fe II) and absorption lines, which are close to one another. Nevertheless, this fact is not well established, since in our spectra we detected only a few Fe II lines with reliably measured $R V \mathrm{~s}$. In most cases these lines are weak, and their profiles are affected by close absorption lines.

From our $R V$ data, although sparse, we can roughly estimate some parameters of the binary. Since the system is not close to us, the companions are large and the orbital period $\left(P_{\text {orb }}\right)$ cannot be small. The lowest $P_{\text {orb }}$ consistent with our $R V$ data is $\sim 55$ days (see Fig. 5). If we assume that we observed the full $R V$ amplitude (the semi-amplitude $K_{2} \sim$ $45 \mathrm{~km} \mathrm{~s}^{-1}$ ), then one can calculate the secondary's mass function $f\left(m_{2}\right)=0.5 M_{\odot}$. In order to estimate the companion masses, we need to know the inclination angle of the system's rotation axis $(i)$ with respect to the line of sight. It is most likely large (probably even close to $90^{\circ}$ ), because the emission line profiles have a P Cyg type shape. This suggests a large optical depth for bound-bound transitions in the line of sight. Since it is hard to expect a spherical CS envelope in a binary system, we can assume that it is flattened and the plane of preferential matter concentration coincides with the orbital plane. Therefore $\sin i$ should not be far from 1, suggesting the companion masses of $\sim 2 M_{\odot}$ if equal. This is too low for such distant and hot stars.

This consideration prompted us to suggest that $P_{\text {orb }}$ and/or $K_{2}$ are larger. Our calculations of different $R V$ curves 
for both circular and eccentric orbits show that our data set is in the best agreement with $P_{\text {orb }} \sim 175$ days, $K_{2} \sim 55 \mathrm{~km} \mathrm{~s}^{-1}$, and $e \sim 0.6$. Low-eccentricity with such a long period does not agree with the obtained $R V$ data. The best long-period solution gives $f\left(m_{2}\right)=3\left(1-e^{2}\right)^{3 / 2} M_{\odot}$ or $m_{2} \sim m_{1} \sim 6.5 M_{\odot}$.

Such masses still seem too low for such luminous stars as the system's companions. This might be due to an underestimation of the $R V$ amplitude, which must be verified by follow up spectroscopic observations at more orbital phases. Even a small increase in this parameter may raise the companions' masses significantly, as masses $\propto K^{3}$. On the other hand, even an intermediate-mass binary with a short initial orbital period may evolve into an object similar to that of HDE 327083 (cf. van den Heuvel 1994).

We can estimate other parameters of the binary on the basis of the longer-period solution and the secondary's parameters derived in Sect. 3.1 and 4.1. The companions' $T_{\text {eff }}(20000 \mathrm{~K}$ and $7000 \mathrm{~K}$ ) imply bolometric corrections of -2 mag and 0 mag, respectively (Miroshnichenko 1998a). This gives bolometric luminosities $M_{\text {bol }}=-7.8 \pm 1.3 \mathrm{mag}\left(\log L / L_{\odot}=5.0 \pm\right.$ $0.4)$ for the primary and $-5.8 \pm 1.3 \mathrm{mag}\left(\log L / L_{\odot}=4.2 \pm 0.4\right)$ for the secondary. Therefore, the radii would be $27 \pm 15 R_{\odot}$ for the primary and $86 \pm 32 R_{\odot}$ for the secondary. Assuming that the total mass of the binary is $\sim 20 M_{\odot}$, one can derive the orbital semi-major axis $a \sim 360 R_{\odot}$ or 1.7 A.U. The mass does not crucially affect this parameter, since it is proportional to $a^{1 / 3}$. The companions' separation, even at periastron with $e \sim 0.6$, is consistent with their size estimates. On the other hand, an evolved long-period binary is likely to have a less-eccentric orbit due to circularization. The latter suggestion is supported with small intensity variations of the emission-line spectrum. At the same time, the estimated orbital and stellar parameters suggest that the system may experience mass-exchange at least at phases near periastron.

\subsection{The CS matter}

The emission-line profiles contain information about the CS matter distribution in the system. Most of them have P Cyg types, indicating the presence of a high opacity region in front of the hot companion. At the same time, the line absorption components are centered at two different $H R V \mathrm{~s}\left(\sim-200 \mathrm{~km} \mathrm{~s}^{-1}\right.$ for the He lines and $\sim-120 \mathrm{~km} \mathrm{~s}^{-1}$ for the Balmer lines, see Fig. 6). The absorption components of the Fe II lines blueward of $\lambda \sim 5300 \AA$ have $H R V \mathrm{~s}$ of $\sim-120 \mathrm{~km} \mathrm{~s}^{-1}$. As mentioned in Sect. 3.1, they become weaker in the redded Fe II lines, and their $R V$ are difficult to measure. This effect is most likely due to the increasing contribution of the secondary companion. Also, like the He I lines, the entire Fe II line profiles follow the primary's orbital motion. The only Balmer line $(\mathrm{H} \alpha)$ exhibits a double-peaked profile with the blue emission peak located at virtually the same $R V$ as that of the He I absorption component. Also the Fe II lines and the Ca II IR triplet show double-peaked emission-line profiles with the peak separation $\sim 3$ times smaller than that in the $\mathrm{H} \alpha$ line.

Since the He I lines have the highest excitation potential among the considered species, they seem to be formed in the most inner region of the primary's envelope. The Balmer lines are usually formed throughout the entire gaseous envelope, while the Fe II and Ca II lines with even lower excitation potentials are formed in the envelope's outer parts (at least their emission components).

The described variety of the line profiles suggests a velocity distribution decelerating outwards from the stellar surface rather than an accelerating one, usual for early-type supergiants. For example, in the spectrum of $\mathrm{P} \mathrm{Cyg}$, a supergiant with a slowly accelerated mostly spherical wind, absorption components of the He I lines are located at lower $R V \mathrm{~s}$ than those of the Balmer lines (Najarro et al. 1997). The P Cyg type profiles indicate that the envelope is not very flattened, otherwise double-peaked profiles centered at the star's position would be observed. The absorption components of the Fe II lines may be formed in this part of the envelope. The double-peaked shape of the emission components of the Fe II and $\mathrm{Ca}$ II lines may indicate that the envelope becomes flatter at large distances from the primary.

The presence of the blue emission peak in the $\mathrm{H} \alpha$ profile suggests that there is an emitting region near the stellar surface. The problem is why such a peak is not observed in the other Balmer lines. This might be due to a high density at the envelope's base responsible for a steep decrease of the higher level populations, or to the CS dust in front of the star absorbing the blueshifted line emission. Both these possibilities might work together. Summarizing all the information about the emission line profiles, we can conclude that the primary's CS envelope: a) is dense, so that forbidden lines are extremely weak; b) is optically-thick in most of the observed transitions; and c) has a height scale and velocity that decrease outward from the stellar surface.

There are no signs of line emission originating in the vicinity of the secondary companion. This suggests that there is much less CS matter around it than around the primary. There may be two major sources of the CS matter in the system: the primary's stellar wind and the matter transfer from the secondary, especially if it fills its Roche lobe at least at periastron. Since we do not see the primary's photospheric lines even in spectra with high SNR, the star may be a fast rotator, so the lines are shallow. Our suggestion about a high velocity at the envelope's base would agree with this picture. The primary could be additionally spun up through angular momentum transfer from the secondary's material. The decelerating outwards velocity distribution may explain why the Balmer line profile modeling presented by Machado et al. (2001), who employed an accelerating wind kinematics, was not successful. We do not attempt to model the line profiles here, because this task requires a thorough theoretical investigation of the radiation transfer in a complex CS medium. More high-resolution and high SNR spectroscopy than we have is needed to further constrain the latter's properties.

\section{Conclusions}

Our spectroscopic observations of a southern emission-line object HDE 327083 obtained in 2000-2002 resulted in the following findings, most of which have not been reported before. 
1. Numerous absorption lines of neutral metals were discovered in the object's spectrum. They appear to belong to the photosphere of a moderately cool star $\left(T_{\text {eff }} \sim 7000 \mathrm{~K}\right)$, suggesting that HDE 327083 is a binary system rather than a single early B-type star.

2. The $R V$ variations, detected in both emission and absorption lines, vary in antiphase. The variations seem to be regular with an observed amplitude of $\sim 80 \mathrm{~km} \mathrm{~s}^{-1}$ for both types of lines. The period is not tightly constrained due to the data sparseness. However, the companions' high luminosities (and hence radii) favor the larger of possible periods ( $\sim 6$ months), found by fitting of the absorption $R V$ curve. This discovery strongly supports the system's binary nature.

3. The best fit $R V$ curve parameters imply a secondary mass function $f\left(m_{2}\right)=3\left(1-e^{2}\right)^{3 / 2} M_{\odot}$. Under the assumptions that the companions' masses are equal and that the orbit is seen edge-on, the system should have a relatively large total mass $\left(\sim 25 M_{\odot}\right.$ at $\left.e=0\right)$. This does not contradict existing models of the binary system evolution and suggests that initially HDE 327083 was a short-period $\beta$ Lyrae type system (van den Heuvel 1994).

4. The observed spectral line content and the SED suggest that the hot companion (primary) has an early B spectral type (most likely B2 \pm 1 ), while the cool companion (secondary) is an F-type star. The companions have similar $V$-band brightnesses. The primary seems to be a rapidly rotating star $\left(v \sin i \sim 200 \mathrm{~km} \mathrm{~s}^{-1}\right)$. Modeling of the photospheric lines suggests the following secondary parameters: $T_{\text {eff }} \sim 7000 \mathrm{~K}, \log g \sim 2.0$, and $v \sin i \sim 40 \mathrm{~km} \mathrm{~s}^{-1}$.

5. The IS features detected in the spectrum of HDE 327083 indicate that it is located within the Sagittarius spiral arm at a distance $D \sim 1.5 \pm 0.5 \mathrm{kpc}$. The primary's luminosity (if the same as that of the secondary) is then much lower than was previously thought (-10 mag, Lopes et al. 1992).

6. The CS matter is most likely located mainly around the primary, which seems to be the only source of the detected emission lines in the system. Most of the emission lines have P Cyg type profiles. The exception is $\mathrm{H} \alpha$, which shows an additional blue emission peak at a $R V \sim-200 \mathrm{~km} \mathrm{~s}^{-1}$. The peak's position coincides with that of the absorption components of the He I lines, while the Balmer lines' absorption components are located at a $R V \sim-120 \mathrm{~km} \mathrm{~s}^{-1}$. These data suggest that the CS matter velocity decreases outwards from the primary. Overall, the emission line profiles are consistent with a hypothesis of a mildly flattened CS envelope. The envelope's symmetry plane is most likely viewed nearly edge-on, which also seems to be the orbital plane orientation.

Despite the large progress in understanding of the nature of HDE 327083, a number of issues still need to be clarified by follow up observations. In particular, high-resolution optical spectroscopy is needed to further constrain the orbital parameters. Multicolour photometry is capable of verifying whether the system shows eclipses. High SNR spectroscopic observations at different orbital phases will help to determine the emission-line profiles' fine structure to refine the CS envelope's kinematics and the companions' current mass ratio.

HDE 327083 becomes the fifth binary system among the Be stars with warm dust (the other four are MWC 623, CI Cam, AS 381, and V669 Cep), so that binaries are presently about 1/4 of the total number of these objects (see Miroshnichenko et al. 2002a). This result strengthens the support for our hypothesis that the complicated observed features of the group are due to a binary nature. It also shows the importance and effectiveness of long-term multi-technique observational programs in revealing their true properties.

Acknowledgements. This paper uses observations made at the SouthAfrican Astronomical Observatory (SAAO). We thank T. Lloyd Evans, D. Kilkenny, F. Marang, and F. Van Wyk for obtaining the multicolour photometry of HDE 327083. We are also grateful to the referee M. van den Ancker for his valuable comments. A. M. and K. S. B. acknowledge support from NASA grant NAG5-8054 and thank the IRTF staff for their assistance during the observations. Karen Bjorkman is a Cottrell Scholar of the Research Corporation, and gratefully acknowledges their support. This research has made use of the SIMBAD database operated at CDS, Strasbourg, France.

\section{References}

Allen, C. W. 1955, Astrophysical Quantities (London, University of London: Athlone Press)

Allen, D. A., \& Swings, J.-P. 1976, A\&A, 47, 293

Carlson, E. D., \& Henize, K. G. 1979, Vistas in Astronomy, 23, 213

Conti, P. 1997, in Luminous Blue Variables: Massive Stars in Transition, ed. A. Nota, \& H. J. G. L. M. Lamers, ASP Conf. Ser., 120,161

He, L., Whittet, D. C. B., Kilkenny, D., \& Spencer Jones, J. H. 1995, ApJS, 101, 335

Henize, K. G. 1952, ApJ, 115, 133

Herbig, G. H. 1993, ApJ, 404, 142

Hubeny, I., Lanz, T., \& Jeffery, C. S. 1995, Synspec - A User's Guide

Kozok, J. R. 1985, A\&AS, 61, 387

Klochkova, V. G., Yushkin, M. V., Chentsov, E. L., \& Panchuk, V. E. 2002, Astron. Rep., 46, 139

Kurucz, R. L. 1994, Smithsonian Astrophys. Obs., CD-ROM No. 19

Lopes, D. F., Neto, A. D., \& de Freitas Pacheco, J. A. 1992, A\&A, 261, 482

Machado, M. A. D., de Araújo, F. X., \& Lorenz-Martins, S. 2001, A\&A, 368, L29

McCarthy, J. K., Sandiford, B. A., Boyd, D., \& Booth, J. 1993, PASP, 105,881

McGregor, P. J., Hyland, A. R., \& Hillier, D. J. 1988, ApJ, 324, 1077

Miroshnichenko, A. S. 1996, A\&A, 312, 941

Miroshnichenko, A. S. 1998, in Fundamental Stellar Properties: The Interaction Between Observation and Theory, ed. T. R. Bedding (Publ. School of Physics, University of Sydney, Australia), Proc. IAU Symp., 189, 50

Miroshnichenko, A. S., Chentsov, E. L., Klochkova, V. G., et al. 2000, A\&AS, 147, 5

Miroshnichenko, A. S., Levato, H., Bjorkman, K. S., \& Grosso, M. 2001, A\&A, 371, 600 
Miroshnichenko, A. S., Bjorkman, K. S., Chentsov, E. L., \& Klochkova, V. G. 2002a, in Exotic Stars as Challenges to Evolution, Proc. IAU Coll. 187, ed. C. Tout, \& W. Van Hamme, ASP Conf. Ser., 279, 303

Miroshnichenko, A. S., Klochkova, V. G., Bjorkman, K. S., \& Panchuk, V. E. 2002b, A\&A, 390, 627

Najarro, F., Hillier, D. J., \& Stahl, O. 1997, A\&A, 326, 1117

Neckel, Th., \& Klare, G. 1980, A\&AS, 42, 251

Olnon, F. M., Raymond, E., \& IRAS Science Team 1986, A\&A, 65, 607

Osterbrock, D. E. 1989, in Astrophysics of Gaseous Nebulae and Active Galactic Nuclei (Mill Valey: University Science Books)
Robinson, E. L., Ivans, I. I., \& Welsh, W. F. 2002, ApJ, 565, 1169

Rydgren, A. E. 1974, PASP, 86, 363

Strajzhys, V. 1977, in Multicolour photormetry of stars (Vilnius: Mokslas Publ.)

van den Heuvel, E. P. J. 1994, in Interacting binaries, ed. S. N. Shore, M. Livio, \& E. P. J. van den Heuvel (Berlin: Springer-Verlag), 372

Whitelock, P. A., Feast, M. W., Roberts, G., Carter, B. S., \& Catchpole, R. M. 1983, MNRAS, 205, 1207

Zickgraf, F.-J., Wolf, B., Stahl, O., Leitherer, C., \& Klare, G. 1985, A\&A, 143, 421

Zickgraf, F.-J. 2001, A\&A, 375, 122 